\title{
Ein Epigone bekennt sich zu den Herausgebern der Gesamtausgabe der Werke von Friedrich List und sieht sich in deren Nachfolge
}

\section{Eugen Wendler}

Online publiziert: 20. April 2021

(C) Der/die Autor(en) 2021

Zusammenfassung Seit 5 Jahrzehnten steht die Erforschung von Leben, Werk und Wirkungsgeschichte von Friedrich List (1789-1846) im Zentrum der wissenschaftlichen Arbeit von Eugen Wendler. Im Laufe der Zeit sind ca. 30 Monographien und eine größere Anzahl von wissenschaftlichen Aufsätzen und journalistischen Artikeln entstanden. Dabei baute Eugen Wendler auf der unschätzbaren Vorarbeit der Herausgeber der Gesamtausgabe von Lists Werken von 1925 bis 1935 auf.

Der vorliegende Aufsatz vermittelt einen Überblick über die Buchpublikationen von Eugen Wendler zur List-Forschung. Mit seinem eindrucksvollen Oeuvre bekennt er sich zum letzten lebenden Fossil in der Nachfolge der FLG und erweist damit den Herausgebern die gebührende und längst überfällige Wertschätzung und Achtung.

Schlüsselwörter Beiträge zur Listforschung von E. Wendler zwischen 1971 und 2021 - Die globale List-Rezeption · List als Vordenker der europäischen Integration und der Sozialen Marktwirtschaft · Die Entwicklungstheorie von F. List und die Dritte Welt · Das US-amerikanische Exil von F. List

E. Wendler $(\bowtie)$

Hochschule Reutlingen, Reutlingen, Deutschland

E-Mail: eugen-wendler@web.de 


\title{
An Epigone is committed to the editors of the complete edition of the works of Friedrich List and sees himself in their succession
}

\begin{abstract}
For 50 years, the knowledge of life, work and history of effects of Friedrich List (1789-1846) have been at the centre of Eugen Wendler's scientific work. Over time, about 30 monographs and a large number of scientific essays and journalistic articles have been created. In doing so, Eugen Wendler built on the priceless work of the editors of List's works from 1925 to 1935 and continued it.

The present article provides an overview of Eugen Wendler's books published on List. With his impressive oeuvre, he acknowledges himself as the last living fossil in the succession of the FLG and pays them the due and long overdue respect and appreciation.
\end{abstract}

\section{Vorbemerkung}

In seinem Beitrag über die „Erinnerung an die ehemalige Friedrich List-Gesellschaft (FLG) - Würdigung ihres Initiators Edgar Salin sowie der anderen Herausgeber der Gesamtausgabe von Lists Schriften/Reden/Briefen“" (Reminder of the former Friedrich List-Gesellschaft (FLG) - appreciation of its initiator Edgar Salin as well as the other editors of the complete editions of List's writing/speeches/letters) legt der Autor dar, wie er zur List-Forschung kam. Nach der ersten Berührung hatte er festgestellt, dass die Kenntnis von List sowohl in Fachkreisen als auch in der breiten Öffentlichkeit, einschließlich seiner Heimatstadt Reutlingen, erschreckend gering, ja man kann sagen, praktisch gleich null war. Außerdem hatte er erkannt, dass die wenigen ,wissenschaftlichen Fachaufsätze“, die er damals zu Rate ziehen konnte, im Wesentlichen auf lexikalische Kurzbiographien und die unkritische Aussage beschränkt waren, dass List Eisenbahnpionier und Protektionist gewesen sei.

Diese Erkenntnis führte ihn zur Überzeugung ganz von vorne, d.h. bei Lists Biographie anfangen zu müssen, um auch für sich selbst einen Zugang zum „Phänomen List" zu verschaffen. Weil er davon ausging, dass sich viele flüchtigen Leser nur einen groben Überblick über das Leben und Wirken von List verschaffen wollen und eine komprimierte, lesefreundliche Abhandlung mit anschaulichem Bildmaterial bevorzugen, schwebte Eugen Wendler für den Anfang eine Bildbiographie mit relativ wenig Text vor. Das Ergebnis war das Buch „Friedrich List - Leben und Wirken in Dokumenten“, das 1976 im Verlag Oertel+ Spörer in Reutlingen erschienen ist.

Über den vom Verlag bestimmten Titel ist der Verfasser nie glücklich gewesen, weil dieser eher auf eine trockene wissenschaftliche Sammlung von historischen Dokumenten, als auf eine flüssige, unterhaltsame und allgemein verständliche Darstellung hindeutet, wie sie für das breite Publikum bestimmt war. In einer Buchbesprechung hat Klaus Dreyhaupt von der Ruhr-Universität in Bochum den Bildband in den Mitteilungen der List Gesellschaft (LG) rezensiert und bemerkt: „,Wer ohne größeren Zeitaufwand sein Wissen über List ergänzen will und sich dabei nicht mit der stenographischen Kürze lexikalischer Darstellungen begnügt, wird das Erscheinen dieser recht geglückten Synthese aus Kurzbiographie und Bilddokumentation begrüßen. Die Kurzbiographie wendet sich vor allem an die Leser, die eine Ein- 
führung in Person und Werk des Mannes suchen, dessen Name unsere Gesellschaft trägt. In Verbindung mit der Bilddokumentation vermag sie aber auch das Interesse solcher Leser finden, die - nachdem sie bereits mit Lists theoretischer Arbeit und seinen politischen Vorschlägen vertraut sind, - ergänzend sein persönliches Schicksal kennenlernen wollen. Der sowohl inhaltlich, wie auch in der äußeren Aufmachung gelungene Bildband kann somit unseren Mitgliedern zur Lektüre besonders empfohlen werden."

Vielleicht hätte man noch hinzufügen können, dass der Bildteil eine Vielzahl von bisher unbekannten Abbildungen enthält, die damals mühsam zusammengetragen und die Bildrechte teuer bezahlt werden mussten, um den tragischen Lebensweg von List anschaulich zu illustrieren. Die erste Beschäftigung mit dem Leben und Werk von List hat dann bei E. Wendler den Grundstein für die weitere intensive wissenschaftliche Auseinandersetzung gelegt, von der er damals nicht ahnen konnte, dass sie sich über 5 Jahrzehnte hinweg erstrecken und ihn durch viele Höhen und Tiefen führen wird.

\section{Die wissenschaftliche Erforschung und Auseinandersetzung mit dem Werk von List bis zur Gründung des Friedrich-List-Instituts (FLI)}

Nachdem Eugen Wendler zum 1. Mai 1972 als Dozent für Betriebswirtschaft an die neugegründete Fachhochschule Reutlingen berufen wurde und sich dadurch die Aussicht auf eine akademische Karriere eröffnete, wollte er zunächst seine Promotion nachholen. Deshalb stellte er sich die Frage, ob in Lists Werk auch Ideen und Ausführungen zur Betriebswirtschaftslehre - damals Privatwirtschaftslehre genannt - zu finden sind. Das Ergebnis dieser Untersuchung war eine Arbeit über „Das betriebswirtschaftliche Gedankengebäude von Friedrich List - Ein Beitrag zur Geschichte der Betriebswirtschaftslehre“, mit der er an der Universität Tübingen zum Dr. rer. pol. promoviert wurde. Er verdankt dies seinen beiden Doktorvätern Alfred E. Ott und Karl-Erich Born, welche die Arbeit betreut, unterstützt und zugleich für die weiteren wissenschaftlichen Ambitionen von E. Wendler den Grundstein gelegt haben.

Im Jahre 1983 hat er dann die „Reutlinger Lebensbilder“ ins Leben gerufen. Die erste Schrift war „Reutlingen und Friedrich List“ gewidmet. Dabei ging es ihm vor allem darum, den großen, aber weitgehend vergessenen Sohn der Stadt stärker im Bewusstsein der Einwohner, insbesondere der Schulabgänger, zu verankern und deutlich zu machen, wie List durch seine politische Agitation die Öffentlichkeit in den deutschen Territorialstaaten des 19. Jahrhunderts wachrütteln wollte (Abb. 1).

Da seine von ihm sehr verehrte Schwiegermutter eine Schweizerin aus dem Wallis war und er zur Schweiz immer eine besondere Affinität hatte, motivierte es ihn, das bis dahin unerforschte Exil von Friedrich List in der Schweiz eingehend zu untersuchen. Da er während seines Studiums an der Universität Mannheim sowie im Nachdiplomstudium Pädagogik, Philosophie und Geschichte studiert hatte, war es ihm möglich, mit einer Arbeit über „,Das Exil von Friedrich List in der Schweiz und sein Meinungsbild über die Eidgenossenschaft" an der Universität Konstanz 
Abb. 1 Karikatur zur politischen Agitation von Friedrich List, mit der er den schlafmützigen ,Deutschen Michel“ wachrütteln wollte

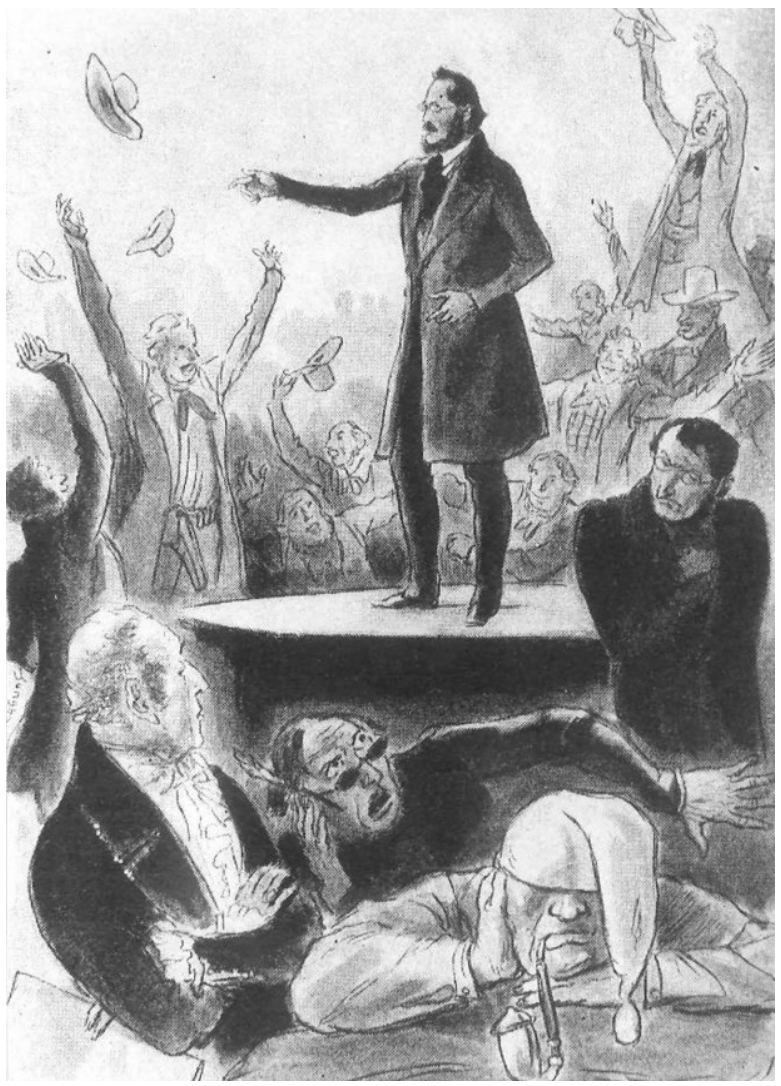

zum Dr. phil. zu promovieren. Auch diesen beiden Doktorvätern, Lothar Burchardt und Bernd Wunder, ist Eugen Wendler mit großem Dank verbunden.

In einer einfühlsamen Buchbesprechung hat Rainer Stöttner, Ordinarius für Volkswirtschaftslehre an der Universität Kassel, die Arbeit als ,erschütterndes Dokument einer Treibjagd“"gewürdigt. Nach der Lektüre dieses biographischen Werkes verliert das „Phänomen List“ vieles von seiner bisherigen Undurchsichtigkeit und Unergründbarkeit. Scheinbare Ungereimtheiten seines Charakters klären sich auf und böswillige Deutungen der Handlungen und Absichten Lists werden als solche entlarvt. In der mit außerordentlicher Sorgfalt und mitunter kriminalistischem Spürsinn durchgeführten Untersuchung sei es dem Autor trotz schwieriger Quellenlage gelungen, diese weithin unbekannte Station im Leben Lists durch Fakten zu belegen und daraus die vielfältige Bedeutung abzuleiten, die Lists erstem Exil für seinen weiteren Lebensweg zukomme. Jeder wissenschaftliche Biograph kenne die ebenso gefährliche wie verführerische Grauzone zwischen eindeutig belegbaren Fakten und lückenfüllenden Vermutungen und Interpretationen. Auf letztere ganz zu verzichten, sei schlechterdings unmöglich, wolle man nicht eine ganzheitliche Würdigung der zu ergründenden Persönlichkeit von vornherein in Frage stellen. Wendler sei es hoch anzurechnen, dass er die Schnittstelle zwischen Fakten und Deutung stets 
klar erkenntlich gemacht habe. Überhaupt werde mit zwangsläufig immer subjektiven Deutungen sehr sparsam umgegangen und dort, wo sie unumgänglich sind, der Leser so breit informiert, dass er deren Plausibilität eigenständig überprüfen könne. Noboru Kobayashi, der Nestor der List-Forschung in Japan hat auf der Mitgliederversammlung des „Vereins für deutsche Wirtschaftsgeschichte“ in Tokyo über diese Arbeit referiert und einen 20seitigen Aufsatz in der „History of Economic Thought“ publiziert.

Im Oktober 1983 nutzte E. Wendler eine Studienreise nach Paris, um im Archiv des „Institut de France“ nach Lists verschollener zweiter Preisschrift zu suchen. Es war zweifelhaft, ob es überhaupt eine zweite „Preisschrift“ gegeben hat. Zumindest galt das Manuskript als verschollen. Wendler war jedoch fest davon überzeugt, dass es existiert und hatte bei seiner Suche auch Erfolg. In dem in französischer Sprache verfassten Manuskript mit dem Titel „Le monde marche“ behandelt List die Frage, welche mutmaßlichen Auswirkungen die Eisenbahnen auf die Wirtschaft, das bürgerliche Leben, den sozialen Zustand und die Macht der Nationen haben. Nachdem der Entdecker das Manuskript transkribiert, übersetzt und kommentiert hatte, wurde es 1985 vom Verlag Vandenhoeck und Ruprecht in Göttingen verlegt. Der Text wurde auch ins Chinesische und ins Japanische übersetzt.

In einer Buchbesprechung in den Reutlinger Geschichtsblättern würdigte der damalige Leiter des Stadt- und Listarchivs Heinz-Alfred Gemeinhardt die Neuerscheinung mit den Worten: „Eugen Wendler legt als Ergebnis seiner mühevollen Arbeit den Text des französischen Originals mit der deutschen Übersetzung, ergänzt durch eingehende Erläuterungen, in diesem Band dar. Für den heutigen Leser wird es schon allein beim Lesen der Kapitelüberschriften verblüffend sein, in welchem MaBe sich List mit den künftigen Auswirkungen der kommenden Eisenbahn auf allen Gebieten des öffentlichen Lebens Gedanken machte. Interessant sind auch Lists weitschauende Prognosen, die sich meist bewahrheitet haben. Dem Verfasser und dem Verlag gebührt mein geschätzter Dank für diesen Band, mit dem wesentlich neue Erkenntnisse zur Liste-Forschung beigesteuert worden sind.“

Die Zeitschrift für Württembergische Landesgeschichte von 1987 enthält eine Rezension von Gert Kollmer, dem damaligen Leiter des Wirtschaftsarchivs an der Universität Hohenheim. „Dem Herausgeber gelingt es in sehr gründlicher und anschaulicher Weise, dem Leben und Wirken Friedrich Lists Einblick zu geben sowie über die Auffindung, Identifizierung, Beschreibung des Manuskripts, die geistesgeschichtliche Einordnung und inhaltliche Bewertung der Preisschrift, die Aufgabenstellung der Preisfrage und das Preisrichterkollegium zu informieren. Der Herausgeber präsentiert sowohl den französischen Originaltext sowie eine gut lesbare und verständliche deutsche Übersetzung. Dabei kann es nur als angenehm empfunden werden, wenn der Herausgeber sich so eng wie möglich an den Originaltext anlehnt; an manchen Stellen aber, in denen Unklarheiten entstehen könnten, eine freie Übersetzung vorzieht.“

In einem Aufsatz im List Forum von 1986 setzte sich Anton P. Müller von der Universität Erlangen-Nürnberg mit der Bedeutung der Preisschrift auseinander. Unter dem Titel „List als Vorläufer der Transaktionskosten-Ökonomik: Zur jüngst entdeckten Preisschrift von 1837“" schreibt er u. a. „Das Manuskript der Preisschrift, dessen Existenz man bisher in Frage gestellt hatte, wurde im Oktober 1983 von Eu- 
gen Wendler im Institut de France entdeckt; die Autorenschaft Friedrich Lists konnte einwandfrei nachgewiesen werden. Es ist nicht nur dogmenhistorisch oder wegen der Klarheit des Urteils und der Weitsicht der Voraussagen interessant. Aus heutiger Sicht erwächst ihre Bedeutung vor allem daraus, dass List in seiner Analyse eine Sichtweise einschlägt, die erst in jüngster Zeit mit dem Begriff ,Transaktionskosten" in der Wirtschaftstheorie thematisiert wird. Klarer noch als in seinem Hauptwerk ,Das Nationale System der Politischen Ökonomie ' erschließt sich in der vorliegenden Schrift das theoretische Konzept der ,produktiven Kräfte ' als Abbau von Transaktionskosten.“

Aus Japan kam ebenfalls eine positive Rückmeldung. Am 21.10.1985 schreibt Noboru Kobayashi: „Ich bin sehr glücklich, dass ich in meinen alten Tagen Lists neu entdeckte zweite Preisschrift lesen kann. Die eingehende Einleitung von Prof. Wendler ist sehr anziehend; er scheint mir der vortreffliche Nachfolger von Artur Sommer zu sein, an den ich liebe Erinnerungen habe“ und am 18.06.1986 berichtet er: „Ich habe eine Besprechung der Preisschrift für die ,History of Economic Thought" vorbereitet; sie wird im Oktoberheft erscheinen und die japanische Gelehrtenwelt über das hohe Niveau der List-Forschung von Eugen Wendler informieren."

Anlässlich des 200. Geburtstages von Friedrich List im Jahre 1989 hatte Wendler die Absicht, in der List-Forschung weitere neue Wege zu beschreiten. Angesichts der wenigen Publikationen, die nach dem II. Weltkrieg zu diesem Thema in der Bundesrepublik und in der DDR erschienen sind, musste er feststellen, dass die Bemühungen der FLG kaum Früchte getragen haben. In der DDR sind damals übrigens mehr Publikationen über List erschienen als in der Bundesrepublik. Allerdings waren diese Werke mehr oder weniger stark ideologisch gefärbt. Sie konzentrierten sich vor allem auf Lists Wirken als Eisenbahnpionier und die Rezeption seiner Ideen durch Karl Marx und Friedrich Engels.

Zum Jubiläumsjahr schwebte Wendler vor, die bis dahin unerforschte internationale List-Rezeption in den Fokus zu nehmen und zu untersuchen, wo und in welcher Weise seine Ideen im Ausland ihren Niederschlag gefunden haben. Dieses Unterfangen hat sich zunächst als unerfüllbare Herkulesaufgabe erwiesen, der er damals nicht gewachsen war. Von den unüberwindlichen Schwierigkeiten, welche diesem Vorhaben entgegenstanden, kann man sich heute kaum noch eine Vorstellung machen. Der eiserne Vorhang war so dicht, dass es praktisch unmöglich war, mit osteuropäischen Wissenschaftlern in Kontakt zu treten und von dort irgendwelche Quellen zu bekommen, von den Übersetzungsproblemen ganz zu schweigen. Es gab noch kein Internet und noch nicht einmal eine Faxverbindung; selbst zu westeuropäischen Wissenschaftlern und fachkundigen Spezialisten in anderen Kontinenten war es schwierig, Kontakte zu knüpfen. Die damalige Spurensuche war extrem mühsam und zweitaufwendig. Außerdem standen weder Forschungsmittel, noch wissenschaftliche Hilfskräfte oder eine Sekretärin zur Verfügung. So dauerte es z. B. über ein Jahr, bis er ein Porträt von dem ehemaligen rumänischen Ministerpräsidenten und List-Anhänger Petre Aurelian von der rumänischen Akademie der Wissenschaften über die rumänische Botschaft oder die Fotokopie von einem MarxAutograph vom Institut für Marxismus-Leninismus der KPdSU in Moskau über die sowjetische Botschaft bekam. Selbst mit Kollegen in der DDR war es trotz eines persönlichen Kontakts mit der damaligen „Hochschule für Verkehrswesen Friedrich 
List in Dresden“ unmöglich, einen Meinungsaustausch zu pflegen. Wenn Eugen Wendler Übersetzungshilfen benötigte, musste er Studenten finden, die ihm halfen und manchmal von ihren Studienaufenthalten im Ausland auch die gewünschten Fotokopien mitbrachten.

Angesichts dieser riesigen Hürden musste er, um sein Vorhaben noch einigermaßen zu retten, unter dem gegebenen Termindruck die ursprüngliche Konzeption quasi in letzter Minute aufgeben, indem er sich mit ca. 20 berühmten Politikern und Staatsmännern des In- und Auslandes begnügte, um an deren Beispiel deutlich zu machen, wie diese Lists Ideen umgesetzt und gewürdigt haben. Außerdem war ihm schon damals wichtig, deutlich zu machen, dass die List-Rezeption in Japan, aber auch in Indien Spuren hinterlassen hat und konnte dafür die in ihren Ländern renommierten Wirtschaftswissenschaftler Prof. Dr. Noboru Kobayashi und Prof. P.R. Brahmananda für eigene Beiträge gewinnen. Da zur damaligen Zeit das Thema „Europäische Union“ im Brennpunkt der politischen Diskussion stand, wollte er darauf aufmerksam machen, welche Visionen Friedrich List in Bezug auf die europäische Integration hatte. Diese in der List-Forschung neuen Ansätze hat er dann in seinem Buch „Friedrich List - Politische Wirkungsgeschichte des Vordenkers der europäischen Integration“ zusammengefasst, das im Oldenbourg-Verlag in München erschienen ist.

Leider ist das Buch nicht nur unter großen Geburtswehen und gewaltigem Zeitdruck entstanden, sondern der Autor erlebte zu dieser Zeit auch einen tiefen physischen und psychischen Einbruch, den man heute als „Burn-out“ bezeichnen würde. Deshalb wurde das Lektorat leider sehr oberflächlich durchgeführt, was für ihn sehr ärgerlich war. Obgleich es aus seiner Sicht eigentlich einen zukunftsweisenden Beitrag zur List-Forschung darstellt, war er mit dem Ergebnis unzufrieden. Aber es stellte immerhin einen ersten Versuch dar, Holzpfähle in ein bisher brach liegendes sumpfiges Terrain einzurammen, auf denen in der Zukunft aufgebaut werden konnte, um deutlich zu machen, in welchen globalen Dimensionen Lists Denken angelegt war (Abb. 2).

Dennoch gab es von dem Bremer Kollegen Dieter Senghaas in der Zeitschrift „Historisch-Politisches-Buch“ eine positive Stellungnahme: „Wendler gehört zu den wenigen fundierten List-Kennern, die ihre eigene Forschung einer gründlichen Erschließung der Biographie Lists widmen. In diesem Buch werden die Reaktionen zeitgenössischer Staatsmänner sowie späterer Würdigungen in der Zeit nach Lists Tod thematisiert. Mit der für 1992 geplanten Verwirklichung des europäischen Binnenmarktes im Rahmen der Europäischen Gemeinschaft gehen Lists kühnste Träume in Erfüllung." Und der Tübinger Historiker Gerd Schulten schreibt in der Zeitschrift „Universitas“: „Eugen Wendler hat so viel neues Quellenmaterial entdeckt, dass Lists Biographie zwar nicht neu geschrieben, aber in vielen Einzelheiten korrigiert und erweitert werden muss. Auf jeden Fall handelt es sich bei Wendlers Buch um eine einzigartige Fundgrube.“ Wenn er hinzufügt: „Weshalb es besonders ärgerlich ist, dass der Text nicht redigiert wurde und nun eine Vielzahl störender Fehler aufweist", muss man ihm leider vollkommen recht geben.

In einer Rezension der „Zeitschrift für Wirtschafts- und Sozialgeschichte“ bemerkt Karl-Heinrich Kaufold aus Göttingen: „Das Buch ist mit großem Fleiß und spürbarer Begeisterung für List und sein Werk erarbeitet worden. Er hebt vieles 
Abb. 2 Friedrich List in mittleren Jahren als Verkehrswirtschaftler

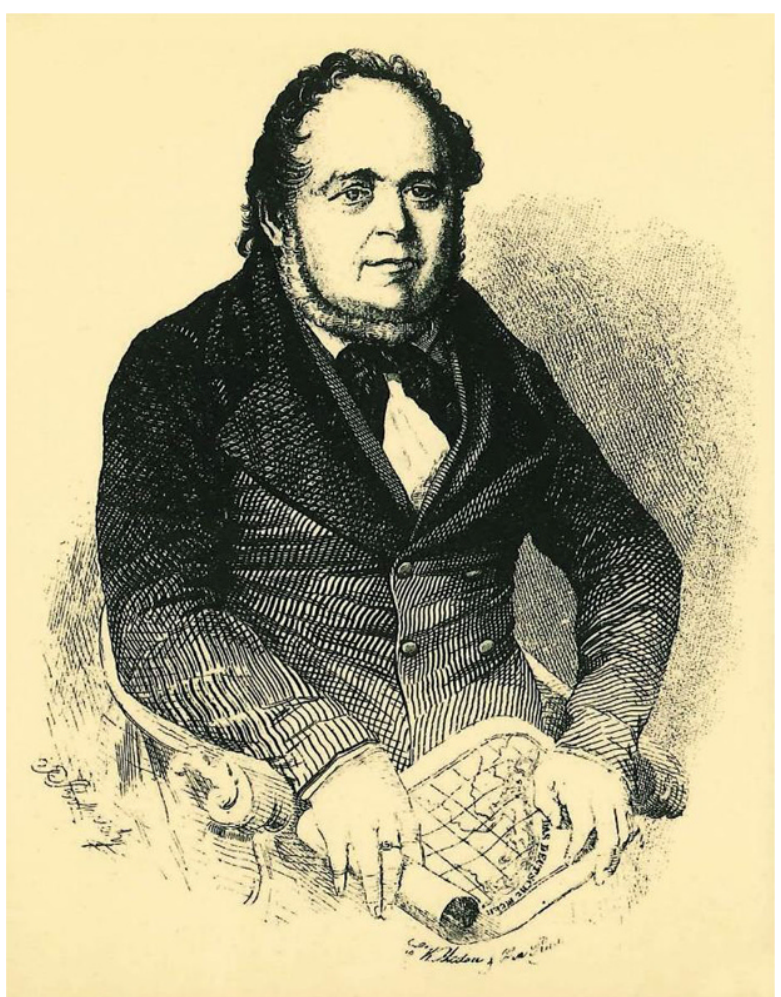

nahezu vergessene ans Licht und bringt vor allem aus vom Verfasser gesammelten Quellen manches Unbekannte. Doch auch Bekanntes gewinnt in der originellen Zusammenstellung des Werkes ein neues Gesicht und Gewicht. Der über rund eineinhalb Jahrhundert reichende, weltumspannende Einfluss List'scher Ideen (freilich auch ihrer nicht seltenen Missverständnisse) wird eindrucksvoll deutlich.“

Der Verlag hat ein Exemplar dem damaligen Bundespräsidenten Richard v. Weizsäcker übersandt, der am 19.06.1989 dem Autor zu dessen Freude und Überraschung antwortete: „Für die liebenswürdige Übersendung Ihres Buches über Friedrich List danke ich Ihnen vielmals. In Ihrem Werk habe ich mit großem Genuss gelesen und dabei wichtige neue Erkenntnisse erhalten. Lists großer und bleibender Beitrag als Vordenker der europäischen Integration verdient ins Gedächtnis gerufen zu werden. Dies ist mit Ihrem Buch vorzüglich gelungen."

Besondere Wertschätzung wurde dem Buch von Noboru Kobayashi zuteil, der im „Journal of Economics“ eine 29seitige Rezension veröffentlichte und dabei das Buch als den Beginn einer neuen Phase der List-Forschung betrachtet; eine Einschätzung, die Eugen Wendler damals noch für überzogen und verfrüht hielt, die sich aber aus der Rückschau betrachtet, bewahrheitet hat. Kobayashi äußerte die Überzeugung: „Wendlers Buch ist insofern richtungsweisend, als es erstmals die politische Wirkungsgeschichte Friedrich Lists systematisch untersucht und somit eine Erklärung 
für seinen bemerkenswerten Nachruhm liefert. Deshalb stellt es einen wichtigen Meilenstein dar, der eine neue Phase in der List-Forschung eingeleitet hat.“

Sein zweiter Beitrag zum List-Jahr war eine zweisprachige Abhandlung zu Lists Exil in den USA, zu der er zahlreiche neue Quellen und unbekanntes Bildmaterial beisteuern konnte. Das Buch hat den Titel: „Friedrich List - ein Pionier auch im deutsch-amerikanischen Bereich - A pioneer in German-American Relations“. Als Verleger für das zweisprachige Werk konnte er den Münchener Verlag Moos + Partner gewinnen. Die Übersetzungskosten hat die Firma Danzer in Reutlingen gesponsert.

Dietmar Kuegler hat über die Publikation im „Magazin für Amerikanistik“ eine Buchbesprechung veröffentlicht und darin lobende Worte gefunden: ,Wer immer sich mit der Geschichte der Amerika-Auswanderung im deutschen Südwesten beschäftigt, wird unvermeidlich auf den Namen Friedrich List stoßen, und wann immer die Namen der bedeutendsten und einflussreichsten Auswanderer in die USA im 19. Jahrhundert genannt werden, ist List dabei. Dies wird in der hervorragenden Arbeit von Professor Wendler umfassend unter Ausleuchtung der Hintergründe, ausgezeichnet illustriert und dokumentiert." In der Bücherecke des Eisenbahn-Journals 11/89 ist zu lesen: „Der in Englisch und Deutsch verfasste Text stammt von einem der besten Kenner des Lebenswerkes von Friedrich List und erstaunt durch eine große Zahl weithin unbekannter Details. Zahlreiche zeitgenössische Stiche illustrieren die sehr empfehlenswerte Schrift.“

Eine Buchbesprechung im Reutlinger General Anzeiger findet ebenfalls positive Worte: „Eine besonders herausragende Buchveröffentlichung zum List-Jahr. Der Autor, Professor für Außenwirtschaft an der Fachhochschule für Technik und Wirtschaft Reutlingen, beleuchtet die Persönlichkeit Friedrich Lists unter dem Aspekt der deutsch-amerikanischen Beziehungen. Sie arbeitet nicht nur Ideengewicht und Weitblick dieses Motors für die industrielle Entwicklung heraus, sondern es gelingt dem Autor auch, die Tragik in Lists Leben, ausgelöst nicht zuletzt durch seine Persönlichkeitsstruktur aufzuhellen. Dabei nutzt der Autor manche blitzende Miniaturszene aus Lists Leben, beispielsweise, wenn er dessen Haft auf dem Hohenasperg beschreibt, um den Stoff griffig und lesefrisch aufzuarbeiten. Die Kapitel über Lists Aufenthalt in den USA - auch sie lebendig durch Einzelheiten mit stark menschlichem Flair - enthüllen gleichzeitig amerikanische Lebensumstände aus der damaligen Zeit. Wendler gewährt in diesen Abschnitten tiefe Einblicke in die Daseinsumstände und Volkswirtschaft. Dabei geht der Autor auch detailliert ein auf die Beziehungen Lists zu bedeutenden amerikanischen Politikern bis hinauf zu Präsidenten.“

Eisenbahnromantiker und Freunde von Modelleisenbahnen wollte Wendler mit einer kleinen Schrift über den Eisenbahnpionier List ansprechen. Leider konnte er für dieses Buch mit dem Titel ,Friedrich List - der geniale und vielverkannte Eisenbahnpionier" nur einen lokalen Verlag finden. Kurt Seidel, ein ausgewiesener Eisenbahnexperte, lobte es in einer Rezension in den Reutlinger Geschichtsblättern und betonte: „Die eisenbahnbezogenen Leistungen eines Friedrich List dokumentarisch und mit wertvollen Quellenhinweisen aufgezeigt zu haben, ist ein besonderes Verdienst von Eugen Wendler. So kann man nicht nur als Eisenbahnhistoriker zur 
Lektüre dieser Arbeit ermuntern, sondern sie auch einem breiten Publikum zum aufmerksamen Studieren empfehlen.“

Das Listjahr 1989 ist für Wendler mit einer Einladung an die „Hochschule für Verkehrswesen“ nach Dresden zu Ende gegangen, wo er auf dem dortigen Symposium über die Entdeckung von Lists zweiter Pariser Preisschrift referieren durfte. Den Höhepunkt der damaligen „List-Ehrung der Deutschen Demokratischen Republik“ bildete der letzte Staatsakt der DDR, an dem er teilnehmen konnte. Kurz darauf brach die DDR zusammen und im List'schen Sinne als „Künder der deutschen Einheit" konnte die deutsche Teilung überwunden und die Wiedervereinigung vollzogen werden.

Dadurch hat sich auch für ihn in Bezug auf die List-Forschung ein neues Betätigungsfeld eröffnet, denn erst nach dem Fall der Mauer war es möglich, ein internationales Netzwerk von Wissenschaftlern aufzubauen bzw. neue Quellen aus dem Ausland zu beschaffen, sodass es möglich wurde, die List-Rezeption in allen europäischen Ländern von Finnland bis Portugal und von Irland bis zur Türkei zu erfassen und in einem Gesamtwerk darzustellen. Um diese Forschung zu institutionalisieren und nicht mehr als Privatissimum erscheinen zu lassen, hat Eugen Wendler 1992 an der Hochschule Reutlingen „Das Friedrich List-Institut für historische und aktuelle Wirtschaftsstudien“ gegründet und bis 2012 geleitet.

\section{Die Fortführung der List-Forschung im Rahmen des List-Instituts der Hochschule Reutlingen}

Am 4. Mai 1992 genehmigte das Wissenschaftsministerium in Stuttgart die Gründung des Friedrich-List-Instituts (FLI). In dem offiziellen Schreiben heißt es: „Das Ministerium für Wissenschaft und Kunst stimmt der Einrichtung eines FriedrichList-Instituts an der Fachhochschule Reutlingen zu. Es wird ausdrücklich darauf hingewiesen, dass der Betrieb des Instituts aus Dritt- und Projektmitteln zu finanzieren ist." Diese Gründung war notwendig, weil sich der Institutsleiter für 1996 gleich drei große Forschungsprojekte vorgenommen hatte, die angesichts des 150. Todestages von Friedrich List und des 100. Todestages von Clara Schumann realisiert werden sollten und unter größten Schwierigkeiten und Hindernissen schließlich auch rechtzeitig abgeschlossen werden konnten.

Zum einen wollte Wendler das angefangene Projekt über die List-Rezeption in allen europäischen Ländern fortführen und zu Ende bringen, zum zweiten die über 300 Briefe und andere Dokumente umfassende Korrespondenz der List-Töchter mit der berühmten Pianistin und Komponisten Clara Schumann und deren Freundes- und Bekanntenkreis sowie drittens eine Sammlung bislang unbekannter Nachrufe zum Tod von Friedrich List herausgeben. Alle drei Projekte waren Mammutaufgaben und noch heute erscheint es rätselhaft, wie er diese neben seinen Lehrverpflichtungen und sonstigen Aufgaben an der Hochschule geschafft hat.

Im Rahmen des ersten Projekts wurden 30 europäische Länder in die List-Rezeption einbezogen. Dazu konnte er für die Hälfte ausländische Fachkollegen gewinnen. Die andere Hälfte hat er selbst recherchiert, was ohne die selbstlose Unterstützung von mehreren Studenten, insbesondre bei der Übersetzung fremdsprachiger Tex- 
te, unmöglich gewesen wäre. Für die Drucklegung konnte er den Verlag Schäffer/ Poeschel in Stuttgart gewinnen. Das Buch mit dem Titel „Die Vereinigung des europäischen Kontinents - Friedrich List - Die gesamteuropäische Wirkungsgeschichte seines ökonomischen Denkens" hat über 600 Druckseiten.

Für das Geleitwort konnte er den damaligen Ministerpräsidenten des Landes Baden-Württemberg Erwin Teufel gewinnen. Dieser schreibt u.a.: „Ich freue mich, dass das vorliegende Werk noch einmal das hervorragende Schaffen und Wirken, die internationale Ausstrahlung und Bedeutung des Reutlingers Friedrich List veranschaulicht. Mein herzlicher Dank gilt den Autoren, allen voran dem Spiritus rector dieses anspruchsvollen Forschungsprojekts, Professor Dr. Eugen Wendler, der viel Zeit und Mühe darauf verwendet hat, die Rezeption der List'schen Ideen fast überall in Europa nachzuweisen. Dank dieses Engagements kommen wir rechtzeitig zum 150. Todestag des berühmten baden-württembergischen Landeskindes in den Genuss der Lektüre, die erneut deutlich macht, dass der Weltbürger List vor allem ein Patriot gewesen ist, der seine Lebensaufgabe darin sah, für eine bessere Zukunft in Württemberg, in Deutschland und Europa zu streiten."

Das Handelsblatt brachte am 12.04.1996 eine Buchbesprechung, in der u. a. hervorgehoben wird: ,Viele bisher unbekannten Quellen werden erstmals erschlossen: Sie belegen einen beachtlichen geistigen und politischen Einfluss von Lists ökonomischem Denken in allen europäischen Ländern. Dabei zeigt sich, dass List ein konsequenter Vordenker der europäischen Integrationsidee war." Weitere Buchbesprechungen sind im Journal of the History of Economic Thougtht (19.2 Fall 1997, S. 315-7), in der Zeitschrift für Württembergische Landesgeschichte (57. Jg., 1998, S. 540 f.) sowie im List-Forum (Bd. 25, H. 3, S. 328-330) zu finden.

Auch das 500 Druckseiten umfassende Buch „Das Band der ewigen Liebe: Clara Schumanns Briefwechsel mit Emilie und Elise List“", das zeitgleich bei Metzler in Stuttgart erschienen ist, hinterließ in der Musikwelt eine ganze Reihe positiver Reaktionen, wobei hier lediglich die Rezension in der von Robert Schumann gegründeten „Neue Zeitschrift für Musik“, Nr. 2 vom März 1997, erwähnt werden soll. Dort heißt es u.a. „Der hier vorgelegte umfangreiche Briefwechsel mit den Schwestern Emilie (1818-1902) und Elise (1822-1893) List, ist insofern bemerkenswert als er fast die gesamte Lebensspanne Clara Schumanns abdeckt. Die Briefe sprechen auf weite Strecken eine ganz andere Sprache als etwa der Briefwechsel mit ihrem geliebten Ehemann Robert. Kaum einmal ergeht sie sich in Schwärmereien, sondern meist berichtet sie nüchtern und geradezu beobachtend über ihre jeweilige Situation. Wenn man die Briefe nacheinander liest, bleibt nicht mehr viel von romantischer Verklärung, von der Künstlerin als Priesterin, sondern es stellt sich eine Ahnung davon ein, welche Kombination aus künstlerischer Überzeugung, Ehrgeiz, Entbehrungen und Zeitökonomie für eine professionelle Karriere einer Frau im 19. Jahrhundert notwendig war. So entsteht insgesamt ein facettenreiches Bild, das auch als Beitrag zum Thema ,Musikgeschichte als Kulturgeschichte gelesen werden kann.“ Ebenso haben die renommierten Musikwissenschaftler Eberhard Stiefel, Karl-Michael Komma und Johannes Roßner diese Edition mit großem Lob bedacht.

Auch das dritte Projekt, die Sammlung von Nachrufen zum Tod von Friedrich List hat sich als sehr ergiebig und umfangreich erwiesen. Zusammen mit dem Leiter des Reutlinger Stadt- und Listarchivs, Heinz-Alfred Gemeinhardt, wurde die Sammlung 
unter dem Titel „Sey ihm die Erde leicht - Nachrufe zum Tode von Friedrich List in den Reutlinger Geschichtsblättern“ (Jg. 1996, S. 9-181) herausgegeben.

Für diese drei Publikationen erhielt Eugen Wendler 1997 den Landespreis für Heimatforschung Baden-Württemberg, der bei einem Festakt in Karlsruhe verliehen wurde.

Diese Arbeiten haben sehr viel Kraft gekostet. Deshalb ist es nicht verwunderlich, dass der Autor dafür einen hohen Preis bezahlen musste. Um die Jahrtausendwende war seine Gesundheit sehr angeschlagen; physische und vor allem psychische Unpässlichkeiten stellten sich ein. Er hat immer stärker unter einer wachsenden Depression gelitten, die darauf zurückzuführen war, dass er seine gesamte wissenschaftliche Arbeit, vor allem die List-Forschung in Frage stellte, wie das streckenweise auch bei Salin der Fall war. Daran konnten auch die Verleihung des Bundesverdienstkreuzes und der Verdienstmedaille der Stadt Reutlingen nichts ändern. Wie bei Edgar Salin nagte auch an Eugen Wendler die Frage, ob sich die ganze Mühe gelohnt hat oder ob es reine Zeit- und Kraftverschwendung war, weil sich kaum jemand für diesen einsamen Pfad der Wissenschaft interessierte. Seine körperlichen und seelischen Beschwerden führten schließlich zu einem Zusammenbruch, der mit einem Herzinfarkt einen dramatischen Tiefpunkt erreichte. Erst die Reha und die Zeit danach brachten ihn langsam wieder auf die Beine und auf die vorgezeichnete Bahn zurück (Abb. 3).

Schließlich fasste Eugen Wendler den Entschluss, die List-Forschung erneut aufzunehmen und die vielen neuen Quellenfund und Erkenntnisse, die er in der Zwischenzeit gewonnen hatte, in einem neuen Buch zusammenzufassen, das 2004 unter dem Titel „Durch Wohlstand zur Freiheit - Neues zum Leben und Werk von Friedrich List" im Nomos Verlag in Baden Baden als Monographie der List-Gesellschaft (LG) erschienen ist. In einem Geleitwort betont der damalige Vorsitzende der LG Dr. Friedrich Jansen: „Auch wenn sich das wissenschaftliche Interesse der LG auf aktuelle Forschungsprobleme verlagert hat, bekennt sie sich zur Tradition der Vorkriegsinstitution. Seit der Neugründung der LG hat sich das List-Bild erheblich erweitert und vertieft. Herr Prof. Dr. Dr. Eugen Wendler, Leiter des Friedrich-ListInstituts für historische und aktuelle Wirtschaftsstudien an der Fachhochschule für Technik und Wirtschaft Reutlingen, hat sich in seiner 30jährigen Forschungsarbeit mit dem Leben und Werk von Friedrich List befasst und das List-Spektrum durch unzählige neue Quellen erweitert. Der Vorstand der LG würdigt diese Bemühungen und wünscht dem Buch die verdiente Resonanz bei den Lesern."

Vier Jahre später (2008) konnte er die Absicht verwirklichen, Lists Hauptwerk „Das nationale System der politischen Ökonomie“ in einer illustrierten Neuausgabe mit einer aktuellen Interpretation ebenfalls als Monographie der LG herauszubringen. Der damalige Schriftführer der LG, Prof. Dr. Karl-Hans Hartwig, hat dazu folgendes Geleitwort verfasst: „Friedrich List gehört unzweifelhaft zu den Klassikern der Nationalökonomie. In Auseinandersetzung mit Adam Smith, David Ricardo und Jean Baptiste Say behandelt List in diesem Werk die Quellen des nationalen Reichtums und schlägt dabei eine Richtung ein, die gerade heute wieder eine hohe Aktualität besitzt. Zu den Voraussetzungen für einen nachhaltigen Wohlstand zählen für ihn nämlich nicht nur die Industrialisierung eines Landes als Initialzündung sowie jene ,Erziehungszölle‘ zum Schutz junger Industrien, die wissenschaftlich höchst umstritten sind und im Zusammenhang mit Lists Werk vorwiegend themati- 
Abb. 3 Friedrich List nach einem Aquarell von Simon Dittrich um 1989; Original in Privatbesitz

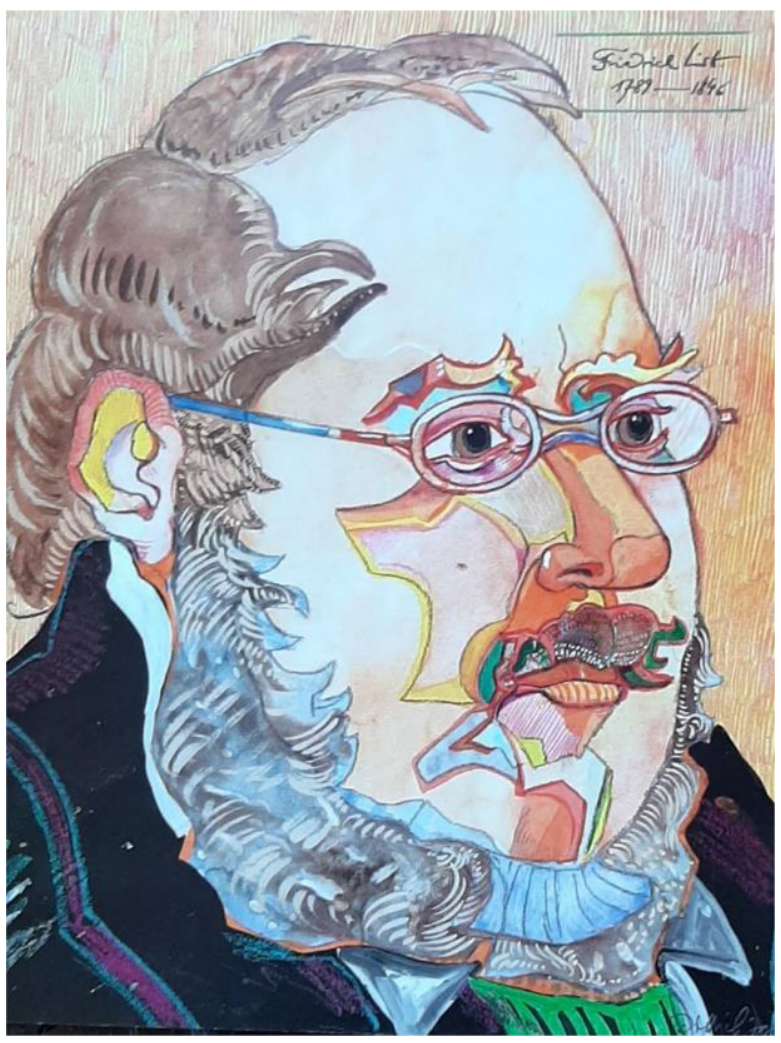

siert werden, sondern dazu gehören auch poltisch-institutionelle Rahmenbedingungen und insbesondere eine gezielte Politik zur Stärkung des heimischen Standorts durch Investitionen in Bildung und in die materielle Infrastruktur.“

Gerade im Humankapital sieht er einen wesentlichen Treiber für das Wachstum und die langfristige Entwicklung von Volkswirtschaften und gerade hier reklamiert er deutliche Unterschiede zu den Ideen der klassischen Nationalökonomie, der er vorwirft, zwar die Bedeutung der Arbeitsproduktivität für den Wohlstand erkannt, nicht aber die Produktivität selbst erklärt zu haben. Betrachtet man die gegenwärtige Diskussion über die Bildungs- und Infrastrukturpolitik in Deutschland, wird deutlich, welche Aktualität die Gedanken von Friedrich List heutzutage besitzen. Die LG möchte mit der Herausgabe einer Neuauflage von Lists Hauptwerk diese Aktualität würdigen und dankt dem List-Forscher Prof. Dr. Dr. Eugen Wendler für seine Zusammenarbeit.

Mit diesen Veröffentlichungen sollte eigentlich Schluss sein. Da der Autor stets große Probleme hatte, für die Publikation seiner Manuskripte einen Verlag zu finden und die jeweils verlangten Druckkostenzuschüsse einzuwerben, wollte er sich diese oft sehr verletzende und desavouierende Quälerei nicht mehr länger antun und deshalb die List-Forschung für sich zum Abschluss bringen. Deshalb wollte er auch 
die Leitung des FLI abgeben, nachdem er 20 Jahre lang heftig dafür gearbeitet und dabei nicht nur die genannten Werke publiziert, sondern darüber hinaus auch noch etliche Vorträge gehalten und viele Aufsätze veröffentlicht hatte.

Insofern war es für ihn eine große Freude, dass er in seinem geschätzten Kollegen, Prof. Dr. Stephan Seiter, einen würdigen Nachfolger finden konnte, mit dem er anschließend nicht nur hervorragend zusammenarbeitet hat, sondern auch freundschaftlich verbunden war. In diesen Freundeskreis wurde auch der Dr. und habil.Vater von Stephan Seiter, Prof. Dr. Harald Hagemann von der Universität Hohenheim, einbezogen. Dabei konnte er zu diesem Zeitpunkt beim besten Willen nicht ahnen, dass ihn auch danach das List-Thema nicht loslassen und schließlich noch zu einer 10bändigen Reihe bei SpringerGabler führen wird.

\section{Die Herausgabe einer 10bändigen List-Reihe im Verlag SpringerGabler in Wiesbaden}

In Zusammenarbeit mit dem renommierten Wissenschaftsverlag SpringerGabler durfte der Autor ab 2013 eine List-Reihe ins Leben rufen, die - was damals überhaupt nicht geplant und abzusehen war - auf 10 Bände anwachsen und damit den krönenden Abschluss seiner List-Forschung, finden wird. Das Überraschende und Bemerkenswerte dabei ist, dass sich die Abfolge der Bände im Nachhinein als schlüssig und folgerichtig erwiesen hat. Diese Reihe wurde nur dadurch ermöglicht, dass der Verlag in großzügiger Weise entgegenkam und auf Druckkostenzuschüsse verzichtete. Dem Verlag war es ein echtes Anliegen, diesem weithin vergessenen Klassiker der deutschen Politischen Ökonomie das ihm gebührende Forum zu verschaffen. Dafür gebührt dem Verlag SpringerGabler Dank und Anerkennung.

Band I trägt den Titel „Friedrich List (1789-1846) - ein Ökonom mit Weitblick und sozialer Verantwortung“ (2013). Er ist der schillernden und tragischen Biographie von List gewidmet. Das Buch enthält unzählige biographische Details, die der Autor im Laufe seiner langjährigen Forschungsarbeit entdeckt hatte und nun das List-Bild wesentlich bereichern. Im Jahre 2014 wurde das Buch ins Englische und 2020 ins Chinesische übersetzt.

Für das Geleitwort konnte der Verfasser den ehemaligen Bundespräsidenten, Prof. Dr. Horst Köhler, gewinnen. Sie haben beide bei Prof. Alfred Eugen Ott promoviert und am 07.07.1977 anlässlich des 500jährigen Jubiläums der Universität Tübingen gleichzeitig das Rigorosum abgelegt. Der frühere Bundespräsident schreibt u.a.: „Dieses Buch lädt dazu ein, Friedrich List wiederzuentdecken und neu zu entdecken für unsere Zeit. Dieses Buch ist selbst schon ein wichtiger Teil einer solchen Neu- oder Wiederentdeckung. Professor Wendler bringt das wissenschaftliche Werk Friedrich Lists zum Sprechen, er setzt sein publizistisches und politisches Schaffen in die vielfältigen zeitgenössischen Zusammenhänge, und er bringt uns Friedrich List als Persönlichkeit nahe als einen Menschen, der auf so vielen Gebieten hervorragend begabt war und dessen Leben doch so oft unter einem Unstern zu stehen schien. Heute, so habe ich aus dem Buch gelernt, wird Friedrich List nicht zuletzt in China gelesen und geschätzt. In Deutschland sind immerhin noch viele Straßen nach ihm benannt. Vor fast sechzig Jahren hat Theodor Heuss, der wie Gustav Stre- 
semann, List sehr geschätzt hat, geschrieben: ,Wenn der Name von Friedrich List genannt wird, bekommen die Deutschen ein schlechtes Gewissen.' Darüber sind die Deutschen hinaus. Sie haben List noch, aber sie kennen ihn nicht mehr. Eugen Wendlers Buch öffnet die Tür für eine Wiederentdeckung oder auch Neuentdeckung mit Fragen, wie zum Beispiel für die Überwindung der Armutsprobleme in Entwicklungsländern und vor allem auch für die Perspektiven der Jugend, die von geradezu verblüffender Aktualität und Relevanz sind. Zeit für List? Ich wüsche dem Buch viele Leser.“

Band II: „Friedrich List im Zeitalter der Globalisierung - Eine Wiederentdeckung“ (2014), beleuchtet die aktuelle Bedeutung von Friedrich List als namhaften Wirtschaftstheoretiker. Hierzu hat der Rektor der Hochschule Reutlingen, Prof. Dr. Hendrik Brumme, ein Geleitwort beigetragen und dabei u. a. betont: „Am 1. Oktober 1971 ist das damalige Hochschulgesetz des Landes Baden-Württemberg in Kraft getreten. Darin wurde als neuartiger Hochschultyp die Fachhochschule ins Leben gerufen. Mit dem neuen Gesetz wurde die Staatliche Ingenieurschule in eine Fachhochschule umgewandelt und gleichzeitig der Fachbereich Betriebswirtschaft gegründet. Prof. Dr. Dr. Eugen Wendler war einer von fünf Dozenten, die in den neuen Fachbereich berufen wurden. Bis zu seiner Emeritierung 2004 hat er über einen Zeitraum von 32 Jahren hinweg, seine ganze Kraft in den Dienst der ,Reutlinger Betriebswirtschaft' gestellt und maßgeblich an deren Erfolgsgeschichte mitgewirkt, die 1995 bei dem ersten deutschen Hochschulranking, bei dem die Fachhochschule Reutlingen auf Anhieb den ersten Platz belegte, ihren ersten Höhepunkt erlebte.“

„Von Anfang an widmete sich Eugen Wendler der Wiederentdeckung des Reutlinger Nationalökonomen Friedrich List. Dazu hat er nicht nur 1992 das Friedrich List-Institut für historische und aktuelle Wirtschaftsstudien an unserer Hochschule gegründet, sondern sich auch durch seine vielen Entdeckungen und Publikationen als List-Forscher in nationalen und internationalen Fachkreisen einen Namen gemacht. Das hier vorliegende Buch steht damit in einer langen Reihe beachteter Beiträge Eugen Wendlers zur wissenschaftlichen Diskussion. Für seine Verdienste um die Hochschule Reutlingen hat die ESB Business School ihn neben den Gründervätern der Reutlinger Betriebswirtschaft im Jahre 2010 mit der Namensgebung eines Hörsaales geehrt." Außerdem wurde ihm 2017 die Verdienstmedaille der Hochschule verliehen.

Band III: „Friedrich List - Die Politik der Zukunft“ (2016) unternimmt in Analogie zu Friedrich Engels, der den Band II und III des „Kapitals“ von Karl Marx nach dessen Tod herausgegeben hat, den Versuch, den von List geplanten aber nicht verwirklichten Band II seines Hauptwerkes „Das Nationale System der Politischen Ökonomie“ inhaltlich zu rekonstruieren, der den Titel „Die Politik der Zukunft“ bekommen sollte. Dabei stützte sich der Autor auf zahlreiche bisher unbekannte Aufsätze von List in der ,Allgemeinen Zeitung“ aus dessen letzter Schaffensperiode.

Hierfür konnte Eugen Wendler nochmals Bundespräsident a. D. Prof. Dr. Horst Köhler und Ministerpräsident a.D. Prof. Dr. h.c. Erwin Teufel für ein Geleitwort gewinnen. Horst Köhler würdigt diese Sammlung als ,eine wertvolle Ergänzung der bisher, vor allem in der Gesamtausgabe der List'schen Werke greifbaren Arbeiten. Schon die thematische Bandbreite der Artikel zeigt, wie List seine ,Politik der Zu- 
kunft' aus einer möglichst genauen Betrachtung und Analyse der Gegenwart und der in ihr herrschenden wissenschaftlichen, kulturellen, technischen und politischen Zustände und Strömungen entwickelte. Das legt Fragen nahe, welche Evidenzen von morgen wir heute übersehen, welche bahnbrechenden Ideen sich heute viel zu schwer durchsetzen, welche Wahrheiten heute unsere Schläfrigkeit verpassen. Es ist reizvoll, wofür ein Kopf wie List, heute stritte - für Nachhaltigkeit als neues Paradigma, für Partnerschaft als neues Leitbild der internationalen Beziehungen, für Terms of Trade, die den weniger entwickelten Nationen endlich faire Bedingungen geben, für bundesweites kostenloses High-Speed-Internet als staatliche Aufgabe wohl gar, deren Erfolg unabsehbaren Gewinn verspricht? Darum ist es wissenschaftlich verdienstvoll, die Schriften von Friedrich List zu bewahren, unser Bild von ihm zu vervollständigen und uns einzuladen zur Beschäftigung damit, was er gedacht und gewollt hat."

Ministerpräsident Erwin Teufel hebt in seinem Geleitwort hervor: „Drei Denkmäler wurden Friedrich List gesetzt: das erste durch seine Vaterstadt Reutlingen, das zweite durch den ersten Bundespräsidenten der Bundesrepublik Theodor Heuss und das dritte durch Prof. Dr. Eugen Wendler, der über 40 Jahre über List geforscht hat. Prof. Wendler hat deutlich gemacht, wie aktuell Lists Ideen heute noch sind. Die Soziale Marktwirtschaft mit freiem Wettbewerb eines vom Parlament festgelegten Ordnungsrahmens, basiert auf Lists Gedankengut. ,Das Maß der Wirtschaft ist der Mensch!', sagt Wilhelm Röpke. Dieser Satz könnte auch von Friedrich List stammen. Er würde heute sagen: Das Maß der Politik, der Wirtschaft und Gesellschaft sind die Eigeninitiative des Menschen, die Hilfe zur Selbsthilfe, die Freiheit der Meinung und der Forschung zum Wohle der gesamten Menschheit. Friedrich List war heimatverbunden und ein Weltbürger zugleich. Theodor Heuss nennt ihn den ,Gesandten der deutschen Zukunft!‘ - Eugen Wendler nennt ihn einen ,Vordenker der Sozialen Marktwirtschaft!‘."

In einem weiteren Geleitwort erinnert der damalige Dekan der Wirtschafts- und Sozialwissenschaftlichen Fakultät der Universität Tübingen, Prof. Dr. Josef Schmid, daran: „Lists kritische Bemerkungen zur englischen Wirtschaftspolitik haben nicht nur zeithistorischen Wert. Er postuliert zugleich - entgegen dem liberalen Credo die aktive Rolle des Staates. Besonders markant für List ist die Betonung des technischen Fortschritts als Mittel zur wirtschaftlichen Entwicklung und der Mehrung des Wohlstandes. Er kritisierte die ,Agriculturisten', plädierte für die Förderung der Eisenbahn und Dampfschifffahrt - ja er entwirft geradezu Ansätze zur ,Science Fiction'. Friedrich List war jedoch nicht lange Professor in Tübingen und die Zeiten, wie auch die Art der wirtschaftswissenschaftlichen Analyse, haben sich grundlegend geändert. Insofern gibt es auch keine Tübinger List-Schule der politischen Ökonomie - aber mit Eugen Wendler - einen beharrlichen Erinnerer.“

Band IV: „Friedrich List: Politisches Mosaik“ (2017) enthält eine Fülle bisher unbekannter Dokumente und Briefe, die das List-Verständnis wesentlich erweitern und bereichern. In einem Geleitwort führt der Ehrenpräsident der European Society for the History of Econmic Thought (ESHET), Prof. Dr. Harald Hagemann, u. a. aus: „Der nimmermüde Elan, mit dem List für den wirtschaftlichen und technologischen Fortschritt kämpfte, zeichnet auch die Forschungen von Prof. Dr. Dr. Eugen Wendler aus, der sich seit fast einem halben Jahrhundert mit Leben, Werk und Wirkungs- 
geschichte dieses großen theoretisierenden Praktikers beschäftigt. Von besonderem Interesse sind Wendlers fundierte Zurückweisung des Vorwurfs imperialistischen Denkens in der Politischen Ökonomie von List sowie seine erneute Auseinandersetzung mit der Rezeption von Lists ökonomischen Theorien, ,der listigen Theorie“ durch Karl Marx und Friedrich Engels, die er anhand der Neuedition der MarxEngels-Gesamtausgabe MEGA vornimmt. Wer die Leidenschaft dieses Forschers für sein Sujet und die Rastlosigkeit kennt, die Wendler mit List als Vorkämpfer der Industrialisierung sowie des technischen und damit auch des gesellschaftlichen Fortschritts teilt, ist überzeugt, dass er bereits kräftig dabei ist, weitere Mosaiksteine für den nächsten wertvollen Band zur List-Forschung zusammenzutragen."

Band V: „Friedrich List - Vordenker der Sozialen Marktwirtschaft“ (2018), positioniert List in die Reihe der großen Ökonomen zwischen Adam Smith und Karl Marx. Dazu hat der ehemalige Präsident des Ifo-Instituts, Prof. Dr. Hans-Werner Sinn, ein Geleitwort verfasst, in dem er u. a. hervorhebt: „Seit 30 Jahren vertritt Eugen Wendler konsequent die These, dass dieser Ökonom mit Weitblick und sozialer Verantwortung, auch als Vordenker der Sozialen Marktwirtschaft zu bezeichnen sei. Er stellt ihn damit in die erste Reihe der nationalökonomischen Klassiker zwischen Adam Smith und Karl Marx. In dem vorliegenden Buch wird diese These nicht nur eindrucksvoll untermauert, sondern auch die aktuelle Bedeutung von Lists breitgefächerten Ideen und Visionen deutlich gemacht. Diese werden in einem spannenden Kontext mit der geheimdienstlichen Observierung verknüpft, der List im Rahmen der Demagogenverfolgung, vor allem durch den habsburgischen Hof und Staatskanzler, Fürst v. Metternich, ausgesetzt war.“ (Abb. 4).

Band VI „Friedrich List und die Dritte Welt“ (2019), behandelt Lists Ideen zur Entwicklungspolitik der wirtschaftlich und politisch nachhinkenden Länder, insbesondere in der südlichen Hemisphäre. Kurz vor seinem Tod hat der Bundesminister a. D. und Vizekanzler Dr. Klaus Kinkel das Buch in einem Geleitwort gewürdigt und dabei betont: „Theodor Heuss war übrigens ein großer Bewunderer von Friedrich List. Er hat 1946 anlässlich des 100. Todestages von List die Gedächtnisrede in der Reutlinger List-Halle gehalten und in weiteren journalistischen Beiträgen dessen liberale Verdienste gewürdigt. Das vorliegende Werk von Eugen Wendler knüpft an diese liberalen Traditionen an, zu denen auch der Ordo-Liberalismus der Freiburger Schule zählt. Auf diesen Grundlagen basiert das System der Sozialen Marktwirtschaft, zu dem Friedrich List als Vordenker einen wichtigen Beitrag geleistet hat. Ebenso visionär sind seine Ideen zur Entwicklungspolitik, die so aktuell sind, dass sie in der Gegenwart auf viele Länder der Dritten Welt übertragen werden können.“

In einem zweiten Geleitwort hat auch Prof. Dr. Dr. h.c. Dieter Senghaas von der Universität Bremen das Buch positiv bewertet: „Eugen Wendler hat sein ganzes Lebenswerk der Biographie sowie den breit gefächerten und vielschichtigen Ideen von Friedrich List gewidmet, und es gibt weltweit keinen zweiten Kenner, der den intellektuellen Werdegang und die politisch motivierten Aktivitäten von List in Europa und in den USA in allen Details aufgearbeitet hat. Erneut wird dieser Sachverhalt durch das vorliegende Buch dokumentiert. Es ist zu wünschen, dass mit der Veröffentlichung der grundlegende Beitrag von List zur Entwicklungsproblematik hinsichtlich seiner Relevanz in der Vergangenheit, Gegenwart und auch noch in der absehbaren Zukunft nun schließlich und endlich sowohl von der Wissenschaft als 
Abb. 4 List-Büste an einem Haus in der Friedrich-ListStraße in Stuttgart

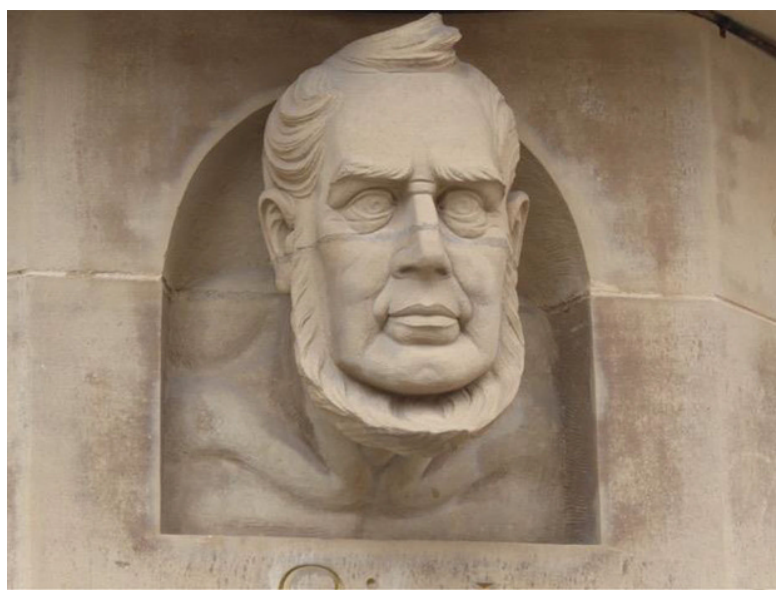

auch von einem interessierten Publikum wirklich wahrgenommen wird. Friedrich Lists Werk ist nicht veraltet. Im Gegenteil! Die vorliegende Monographie erhellt diesen Sachverhalt auf eindrucksvolle Weise.“

Band VII: „Die ethischen Wurzeln der Staats- und Wirtschaftstheorie von Friedrich List“" (2019/20), beleuchtet die philosophischen Grundlagen von Lists Wirtschaftstheorie. In einem Geleitwort von Dr. Wolfang Epp, Hauptgeschäftsführer der IHK Reutlingen, wird lobend hervorgehoben: „Es gibt eine Persönlichkeit, die uns diesen faszinierenden Menschen, der alle Höhen und Tiefen des Lebens mitgemacht hat, bis hin zu seinem bitteren Ende, auf eine eindrucksvolle Weise nahebringt: Professor Eugen Wendler forscht seit über fünfzig Jahren zu Friedrich List. Er ist wie List Reutlinger und seiner Heimatstadt engstens verbunden, ohne auch einmal eine kritische Fußnote zur Stadtpolitik zu unterdrücken. Eugen Wendler hat in dieser Schrift die ethischen Wurzeln der Staats- und Wirtschaftstheorie von Friedrich List akribisch und mit Zeitbezug zusammengetragen. Er führt uns über die Denker der Antike und der griechischen Philosophie über Geistesgrößen der Renaissance bis hin zur Philosophie der Aufklärung und den Protagonisten von Freihandel und Liberalismus. Der Leser ist nicht nur erstaunt, welchen Vordenker und Weitdenker wir in Württemberg und in Reutlingen als eine der größten nennen dürfen. Mein Fazit: besonders lesenswert!“

Dr. Epp ermöglichte es Eugen Wendler, seine in 5 Jahrzehnten zusammengetragene Sammlung von Listiana als Dauerausstellung im „Haus der Wirtschaft“ der IHK Reutlingen zu präsentieren. Sie wurde am 24. Juli 2020 eröffnet. Dafür ist er diesem Förderer und der IHK Reutlingen mit ganz besonderem Dank verbunden.

Band VIII: „Friedrich List - Politische Ökonomie“ (2020), enthält eine Gesamtschau von Lists Ideen zur Wirtschaftstheorie und Wirtschaftspolitik. Hierzu haben der Präsident des Bundesverbandes Deutscher Volks- und Betriebswirte e. V. (bdvb), Willi Rugen und Prof. Dr. Dr. h.c. Adolf Wagner Geleitworte beigetragen. Willi Rugen erinnert daran: „Mit der Gründung des ,Allgemeinen deutschen Handels- und Gewerbsvereins', später umfirmiert zum ,Verein deutscher Kaufleute und Fabrikanten', schuf List eine Form der Interessenvertretung, die man angesichts der 
heutigen, pluralistisch ausdifferenzierten Verbändelandschaft nur als wegweisend bezeichnen kann. Zur Auseinandersetzung mit Lists Gedanken bedarf es der Vermittlung und Einordnung, wie sie durch Eugen Wendler geleistet wird. Mit dem hier vorgelegten Buch - als Zusammenfassung konzipiert, somit auch zur Einführung bestens geeignet - verbindet sich die Hoffnung, dass List in der wissenschaftlichen ebenso wie in der öffentlichen Debatte weiterwirken kann.“

Adolf Wagner beschließt sein Geleitwort wie folgt: „Der humorvolle Alfred E. Ott (1929-1994), Doktorvater von Eugen Wendler, von mir und vielen anderen, würde die großartige forscherische und publizistische Leistung von Eugen Wendler zu Friedrich List mit einem bekannten Ausspruch des Münchener Originals Sigi Sommer (1914-1996) unterstreichen: ,Die Kleinen müssen oftmals jene Leistungen erbringen, die man eigentlich von den Großen erwarten könnte.' Alfred Ott würde sich gewiss , unbändig 'über Eugen Wendlers Leistung freuen, da er eine starke Schwäche dafür hatte, Vergangenes der Lehrgeschichte auf die Zukunft hin zu bearbeiten!"

Band IX :,Friedrich List: Persönliche Begegnungen mit berühmten Zeitzeugen Seine bedeutendsten Gönner und Widersacher" (2020), vermittelt eine eindrucksvolle Synopsis über Lists Bekanntschaft mit historischen Persönlichkeiten, die ihn unterstützt oder auch unbarmherzig bekämpft haben. Hierzu hat ein junger brasilianischer List-Forscher, Prof. Dr. Flávio dos Santos Olivera, ein Geleitwort verfasst und darin u. a. ausgeführt. „Im Zuge der List-Forschung bin ich natürlich auf Prof. Wendler aufmerksam geworden, der als international renommierter List-Forscher besondere Wertschätzung genießt. Seine Publikationen sind für mich eine unerschöpfliche Quelle, um die neuesten Forschungsergebnisse kennenzulernen. Mit Eugen Wendler teile ich die Begeisterung für Friedrich List als ,Ökonom mit Weitblick und sozialer Verantwortung'. Allerdings muss ich gestehen, dass ich in Brasilien auch ein einsamer Rufer in der Wüste bin, weil die Wirtschaftswissenschaft dort genauso, wie in Europa und speziell in Deutschland, auf die anglo-amerikanische Ideologie fokussiert ist. Aber genau so wenig, wie sich Prof. Wendler in seinem langen Forscherleben davon entmutigen ließ, werde ich das tun.“

Band X: „Friedrich List als Eisenbahnpionier in den USA - Eine deutsch-amerikanische Pionierleistung“ (2020), zeichnet in einem Reisebericht Lists Verdienste beim Bau der dritten mit Dampf betriebenen Eisenbahnstrecke in der Neuen Welt, der Little Schuylkill-Railroad in Pennsylvania, nach. Hierzu haben der ehemalige Bürgermeister von Reading PA Tom McMahon sowie der Reutlinger Oberbürgermeister Thomas Keck Geleitworte beigetragen. Tom McMahon führt aus: „Die Entdeckung der Steinkohle in Pennsylvania hatte einen großen Einfluss auf die Lebensbedingungen der Menschen. Der Bau der Little Schuylkill-Eisenbahn, die von Friedrich List und anderen initiiert wurde, beschleunigte den Transport der Kohle und die Energiegewinnung. Prof. Wendler hat einen reichen Schatz an vielen Details zu Tage gefördert und eine eindrucksvolle Dokumentation vorgelegt. Dafür spreche ich ihm persönlich auch im Namen der Bürger der Stadt, meine tiefempfundene Dankbarkeit aus. Ich denke, List wäre stolz auf das, was Prof. Wendler geschaffen hat, um dessen Gedächtnis der Nachwelt zu bewahren."

Und Thomas Keck fügte hinzu: „Ich freue mich, dass Prof. Eugen Wendler nun mit diesem Werk das segensreiche Wirken des gebürtigen Reutlingers Friedrich 
List in Amerika ausführlich darstellt. Ich danke Herrn Prof. Wendler für seinen unermüdlichen Einsatz für Friedrich List und unsere Städtepartnerschaft mit Reading PA.“

Anlässlich seiner Recherche zu diesem Buch wurde Eugen Wendler im Rathaus von Reading 2019 bei einer offiziellen Sitzung des Gemeinderates mit einem Ehrendiplom ausgezeichnet. Bereits 2016 hatte er bei SpringerGabler in Heidelberg eine kleinere Schrift über „Friedrich List's Exile in the United States - New Findings“ herausgegeben.

Alle 10 Bände zeichnen sich durch eine sorgfältige wissenschaftliche Recherche, eine gut verständliche und anschauliche Darstellung und eine reiche Bebilderung aus. Sie sind für jeden ökonomisch Interessierten eine informative und spannende Lektüre und eignen sich auch vorzüglich für Geschenkzwecke.

\section{Einrichtung einer Dauerausstellung im „Haus der Wirtschaft“" der IHK Reutlingen}

Im Laufe seiner Bemühungen um die List-Forschung hat Eugen Wendler alles gesammelt. was an Listiana angeboten wurde. Da dies recht selten der Fall ist, konnte nur eine überschaubare, aber dennoch werthaltige Sammlung entstehen. Im Juli 2020 durfte er den größten Teil dieser Sammlung als Dauerleihgabe im „Haus der Wirtschaft" der IHK Reutlingen, einer reizvollen Jugendstilvilla von 1910, einbringen und für die interessierte Öffentlichkeit zugänglich machen. Dafür ist er der IHK Reutlingen, insbesondere dem Hauptgeschäftsführer Dr. Wolfgang Epp außerordentlich dankbar. Leider wurde der positive Auftakt, den diese Ausstellung in den ersten Wochen gefunden hat, durch die Corona-Epidemie jäh unterbrochen. Deshalb ist zu hoffen, dass sich nach der Normalisierung der Lage das anfängliche Interesse wieder einstellt.

\section{Schlussbemerkung}

Damit betrachtet Eugen Wendler seine zähen Bemühungen um die List-Forschung als abgeschlossen. Er steht nun kurz vor der Vollendung des 82. Lebensjahres und überlässt es der Zukunft, was die Wissenschaft mit seinem Lebenswerk anzufangen weiß. Er fühlt sich als lebendes Fossil und letztes seiner Art in der Tradition der Herausgeber der Gesamtausgabe, denen er mit seinen Arbeiten die verdiente und längst überfällige Reverenz erweisen, sie aus der Vergessenheit herausholen und in ein aktuelles Rampenlicht stellen wollte.

Funding Open Access funding enabled and organized by Projekt DEAL.

Open Access Dieser Artikel wird unter der Creative Commons Namensnennung 4.0 International Lizenz veröffentlicht, welche die Nutzung, Vervielfältigung, Bearbeitung, Verbreitung und Wiedergabe in jeglichem Medium und Format erlaubt, sofern Sie den/die ursprünglichen Autor(en) und die Quelle ordnungsgemäß nennen, einen Link zur Creative Commons Lizenz beifügen und angeben, ob Änderungen vorgenommen wurden. 
Die in diesem Artikel enthaltenen Bilder und sonstiges Drittmaterial unterliegen ebenfalls der genannten Creative Commons Lizenz, sofern sich aus der Abbildungslegende nichts anderes ergibt. Sofern das betreffende Material nicht unter der genannten Creative Commons Lizenz steht und die betreffende Handlung nicht nach gesetzlichen Vorschriften erlaubt ist, ist für die oben aufgeführten Weiterverwendungen des Materials die Einwilligung des jeweiligen Rechteinhabers einzuholen.

Weitere Details zur Lizenz entnehmen Sie bitte der Lizenzinformation auf http://creativecommons.org/ licenses/by/4.0/deed.de. 